\title{
Development and characterization of epoxy nanocomposites based on nano-structured oil palm ash.
}

\begin{abstract}
The aim of this study is to utilize the bio-agricultural waste as filler material for composite production which are abundantly available and low cost compared to the silica, alumina etc. The lacks of sufficient scientific information about the utilization of the oil palm ash (OPA) on composites production were the driving force for the choice of this work. Furthermore, the effect of filler loading percentage on physical, mechanical, thermal and morphology properties of the epoxy nanocomposites were studied. It was concluded that the size of the OPA had been successfully reduced from macromolecular to the nano-size range by high energy ball milling and was confirmed by TEM analysis. The density of the nano-structured OPA filled epoxy composites revealed that increasing filler loading will eventually increase the density. The tensile and flexural strength attained maximum value when the filler loading was 3\%. Also, increase in the thermal stability was observed in case of $3 \%$ filler loading and was attributed to the increase in cross-linking of the epoxy resin in the presence of nanostuctured OPA and having minimum particle to particle interaction and well dispersed nanoparticles.
\end{abstract}

Keyword: Nano-structures; Polymer-matrix composites; Mechanical properties; Electron microscopy. 